\title{
An Optimal Auction with Correlated Values and Risk Aversion*
}

\author{
Péter Esö ${ }^{\dagger}$ \\ MEDS Department, Kellogg School of Management, \\ Northwestern University, \\ 2001 Sheridan Rd, Evanston, IL 60208, USA \\ Running title: An Optimal Auction
}

*I thank Drew Fudenberg and Eric Maskin for very helpful discussions; Márta Eső, Philippe Jehiel, Min Shi, Tomas Sjöström, and Jérôme Vandenbussche for comments. I also thank an associate editor and a referee for insightful suggestions.

${ }^{\dagger}$ Email: eso@northwestern.edu. 


\begin{abstract}
:
We consider an auction setting where the buyers are risk averse with correlated private valuations (CARA preferences, binary types), and characterize the optimal mechanism for a risk neutral seller. We show that the optimal auction extracts all buyer surplus whenever the correlation is sufficiently strong (greater than $1 / 3$ in absolute value), no matter how risk averse the buyers are. In contrast, we note that a sufficiently risk-averse seller would not use a full rent extracting mechanism for any positive correlation of the valuations even if the buyers were risk neutral.
\end{abstract}

Journal of Economic Literature Classification Numbers: D43, D81

Keywords: Optimal Auctions, Correlated Values, Risk Aversion 


\section{Introduction}

The classic single-object auction model (Vickrey [9]) has three important restrictive assumptions: the buyers are risk neutral, and their valuations are purely private and statistically independent. In this note, we simultaneously relax the risk neutrality and independence assumptions and characterize the seller's expected revenue maximizing mechanism in a simple environment.

The two modifications that we consider have already been studied separately. Crémer and McLean [1], [2] found revenue maximizing auctions for correlated values with risk neutral buyers. Maskin and Riley [4] characterized the optimal auction with risk-averse buyers under the assumption that the valuations are statistically independent. By simultaneously relaxing both assumptions we expect to find interesting tradeoffs.

If the buyers' valuations are correlated and they are risk neutral then it is possible to construct an efficient mechanism in which the seller extracts all the information rents from the buyers (see Crémer and McLean [2]). However, this auction form imposes a certain kind of risk on the buyers: their payments are sensitive to the announcements of the others. If the buyers are risk averse, they need to be compensated. This is a previously not analyzed tradeoff between certain screening and insurance incentives.

It is also an interesting theoretical question whether the Crémer-McLean result is an "isolated example." That is, whether full rent extraction is peculiar to the optimal mechanism with risk neutral bidders, or full rent extraction is optimal with any (potentially strong) risk aversion if the signals are sufficiently correlated.

In the next section we introduce a simple auction model exhibiting risk averse buyers with correlated valuations (CARA preferences, two, symmetrically distributed types), and derive the following results. First, we show that the seller's auction design problem is a "regular" screening problem: In the optimal mechanism the incentive compatibility 
constraints bind downward, there is no distortion for the high type (he may only lose to another high type and is fully insured against not winning), and the low type may be rationed and earns no surplus. Second, we show that no matter how risk averse the buyers are, if their types are sufficiently strongly correlated (spefically, if the correlation coefficient is larger than $1 / 3$ in absolute value), then neither type enjoys any surplus. We also show that when valuations are correlated, the seller's revenue is generally nonmonotonic in the degree of the buyers' risk aversion. At the end of our discussion we briefly consider the case of a risk-averse seller. ${ }^{1}$ We note that even if the buyers are riskneutral and hence Crémer-McLean mechanisms could be used, a sufficiently risk-averse seller would prefer not to use such (full-rent-extracting) mechanisms. This is so because such mechanisms provide the seller with high but also highly risky revenue.

Throughout the analysis we make two modelling assumptions. First, we only consider binary types (high or low) and symmetric type-distributions. This assumption makes it possible to parametrize the correlation between the buyers' valuations with just a single number (the correlation coefficient). Second, we model risk aversion by assuming that the bidders' preferences exhibit constant absolute risk aversion. ${ }^{2}$ This assumption rules out random transfers in the optimal mechanism, and makes certain derivations simpler and more intuitive.

\section{The Model}

There are two buyers with private valuations (also called types). A buyer's valuation can take one of two values, either $v^{n}=H$ (high) or $v^{n}=L$ (low), for $n=1,2$. We

\footnotetext{
${ }^{1}$ In the classical (independent private values, risk neutral buyers) setup the case of a risk averse seller is considered by Waehrer et al. [10] and Esö and Futó [3].

${ }^{2}$ In the independent values case, Matthews [5] makes the same assumption.
} 
normalize the seller's commonly known valuation to zero, and assume $H>L>0$.

The joint distribution of the buyers' valuations is symmetric described by the following matrix with $\alpha \in(0,1)$.

$$
\begin{array}{c|c|c|}
\multicolumn{1}{c}{} & \multicolumn{1}{c}{v^{2}=L} & v^{2}=H \\
\cline { 2 - 3 } v^{1}=L & \alpha / 2 & (1-\alpha) / 2 \\
\cline { 2 - 3 } v^{1}=H & (1-\alpha) / 2 & \alpha / 2 \\
\cline { 2 - 3 } & &
\end{array}
$$

That is, $\operatorname{Pr}\left(v^{n}=L\right)=1 / 2$, and $\operatorname{Pr}\left(v^{n}=L \mid v^{-n}=L\right)=\operatorname{Pr}\left(v^{n}=H \mid v^{-n}=H\right)=\alpha$. Note that the coefficient of correlation between the two buyers' valuations is exactly $2 \alpha-1$. We will refer to the entries of this matrix as $\Pi_{i j}=\operatorname{Pr}\left(v^{n}=i, v^{-n}=j\right)$.

The buyers are risk averse, and their von Neumann-Morgenstern utility functions exhibit constant absolute risk aversion (CARA), i.e., $u(w)=-e^{-r w} / r, r>0$. Denote $c_{H}=e^{-r H}$ and $c_{L}=e^{-r L}$, and observe that $0<c_{H}<c_{L}<1$.

The Revelation Principle applies, therefore we can confine our attention to truthtelling Nash equilibria of direct mechanisms. A direct mechanism is given by, for each buyer, his probability of winning conditional on the type-announcements, and his transfers conditional on the type-announcements and a binary variable showing whether he has won the object. Formally, for all $i, j \in\{L, H\}, n \in\{1,2\}$, let $p_{i j}^{n}$ denote the probability of buyer $n$ winning when he announces type $i$ and the other buyer announces type $j$. Also, for all $i, j \in\{L, H\}$ and $n \in\{1,2\}$, let $t_{i j}^{n}$ denote the transfer from buyer $n$ to the seller when the type-announcements are $i$ (from buyer $n$ ) and $j$ (from buyer not $n$ ) and buyer $n$ gets the good; let $\hat{t}_{i j}^{n}$ denote the transfer from buyer $n$ to the seller under the same circumstances but when buyer $n$ does not win. In order to simplify notation we define $y_{i j}^{n}$ and $\hat{y}_{i j}^{n}$, the "disutility factor" corresponding to the transfers, as $y_{i j}^{n}=e^{r t_{i j}^{n}}$ and $\hat{y}_{i j}^{n}=e^{r \hat{t}_{i j}^{n}}$. 


\section{The Optimal Auction}

\subsection{Participation and Incentive Compatibility Constraints}

If the other buyer plays according to the truth-telling equilibrium in an incentive compatible direct mechanism, then buyer $n$ 's expected utility when he has type $i \in\{L, H\}$ and announces $k \in\{L, H\}$ is,

$$
\sum_{j=L, H} 2 \Pi_{i j}\left[p_{k j}^{n} u\left(w+i-t_{k j}^{n}\right)+\left(1-p_{k j}^{n}\right) u\left(w-\hat{t}_{k j}^{n}\right)\right] .
$$

Participation requires that this expression meet or exceed $u(w)$ for $k=i$. Using $\Pi_{i j}=\alpha / 2$ for $i=j$ and $\Pi_{i j}=(1-\alpha) / 2$ for $i \neq j$, the specification of utilities and the notation introduced above, the participation constraints become

$$
\begin{aligned}
\alpha\left(p_{L L}^{n} c_{L} y_{L L}^{n}+\left(1-p_{L L}^{n}\right) \hat{y}_{L L}^{n}\right)+(1-\alpha)\left(p_{L H}^{n} c_{L} y_{L H}^{n}+\left(1-p_{L H}^{n}\right) \hat{y}_{L H}^{n}\right) & \leq 1, \\
(1-\alpha)\left(p_{H L}^{n} c_{H} y_{H L}^{n}+\left(1-p_{H L}^{n}\right) \hat{y}_{H L}^{n}\right)+\alpha\left(p_{H}^{n} c_{H} y_{H H}^{n}+\left(1-p_{H H}^{n}\right) \hat{y}_{H H}^{n}\right) & \leq 1,
\end{aligned}
$$

for types $L$ and $H$ respectively. We will refer to these inequalities (individual rationality for the low and high types) by $\operatorname{IR}_{L}^{n}$ and $\mathrm{IR}_{H}^{n}$, and denote their left-hand sides by $D_{L}^{n}$ and $D_{H}^{n}$, respectively.

Incentive compatibility requires that (1) be weakly greater for $k=i$ than it is for $k \neq i$. That is,

$$
\begin{aligned}
& D_{L}^{n} \leq \alpha\left(p_{H L}^{n} c_{L} y_{H L}^{n}+\left(1-p_{H L}^{n}\right) \hat{y}_{H L}^{n}\right)+(1-\alpha)\left(p_{H H}^{n} c_{L} y_{H H}^{n}+\left(1-p_{H H}^{n}\right) \hat{y}_{H H}^{n}\right) \\
& D_{H}^{n} \leq(1-\alpha)\left(p_{L L}^{n} c_{H} y_{L L}^{n}+\left(1-p_{L L}^{n}\right) \hat{y}_{L L}^{n}\right)+\alpha\left(p_{L H}^{n} c_{H} y_{L H}^{n}+\left(1-p_{L H}^{n}\right) \hat{y}_{L H}^{n}\right) .
\end{aligned}
$$

We will refer to these inequalities (incentive compatibility for the low and high types) 
by $\mathrm{IC}_{L}^{n}$ and $\mathrm{IC}_{H}^{n}$, respectively.

\subsection{Expected Revenue Maximization}

The seller's ex ante expected revenue from buyer $n$ can be written as

$$
\begin{aligned}
R^{n}= & \frac{\alpha}{2 r}\left[p_{L L}^{n} \ln y_{L L}^{n}+\left(1-p_{L L}^{n}\right) \ln \hat{y}_{L L}^{n}+p_{H H}^{n} \ln y_{H H}^{n}+\left(1-p_{H H}^{n}\right) \ln \hat{y}_{H H}^{n}\right] \\
& +\frac{1-\alpha}{2 r}\left[p_{L H}^{n} \ln y_{L H}^{n}+\left(1-p_{L H}^{n}\right) \ln \hat{y}_{L H}^{n}+p_{H L}^{n} \ln y_{H L}^{n}+\left(1-p_{H L}^{n}\right) \ln \hat{y}_{H L}^{n}\right]
\end{aligned}
$$

For a risk neutral seller, the auction design problem is to maximize $R^{1}+R^{2}$ subject to $\mathrm{IR}_{L}^{n}, \mathrm{IR}_{H}^{n}, \mathrm{IC}_{L}^{n}, \mathrm{IC}_{H}^{n}$ for $n=1,2$, and the feasibility constraints, $p_{i j}^{1}+p_{j i}^{2} \leq 1$ for $i, j \in\{L, H\}$. The choice variables are $p_{i j}^{n} \in[0,1]$ and $y_{i j}^{n}, \hat{y}_{i j}^{n} \in(0, \infty)$ for $i, j \in\{L, H\}$ and $n=1,2$.

Since the buyers' preferences are CARA, transfers in the optimal mechanism (conditional on the buyers' announcements and the allocation) are deterministic. This is so because if some $y_{i j}$ 's were random then the seller could replace them by their expected values; this would not alter incentives (as utilities are linear in $y_{i j}$ ), but would lead to a higher expected revenue (as revenue is a concave function of $y_{i j}$ ), which is a contradiction.

The optimal mechanism is symmetric in $n$, that is, $p_{i j}^{1}=p_{i j}^{2}, y_{i j}^{1}=y_{i j}^{2}$, and $\hat{y}_{i j}^{1}=$ $\hat{y}_{i j}^{2}$, for all $i, j \in\{L, H\}$. If it was not then we could define a symmetric ("average") mechanism by

$$
\bar{p}_{i j}^{n}=\frac{p_{i j}^{1}+p_{i j}^{2}}{2}, \bar{y}_{i j}^{n}=\frac{p_{i j}^{1} y_{i j}^{1}+p_{i j}^{2} y_{i j}^{2}}{2 \bar{p}_{i j}^{n}}, \overline{\hat{y}}_{i j}^{n}=\frac{\left(1-p_{i j}^{1}\right) \hat{y}_{i j}^{1}+\left(1-p_{i j}^{2}\right) \hat{y}_{i j}^{2}}{2\left(1-\bar{p}_{i j}^{n}\right)}, \quad n=1,2 .
$$

Since the original mechanism satisfies the IR and IC constraints, this new one satisfies them too (this can be seen by adding the corresponding constraints for $n=1,2$ ), and 
it is clearly also feasible. However, because the seller's objective function is strictly concave in the $y$ 's the new mechanism is strictly preferred by the seller. Therefore, in the remainder of the analysis we will drop the reference to the buyers' identities (the superscript $n)$.

A first inspection of the incentive and participation constraints yields the following result (the proof is relegated to the Appendix).

Lemma 1 In the mechanism that maximizes the seller's expected revenue, from a pair of constraints $\left(I R_{i}, I C_{i}\right)$ at least one is binding. Moreover, either $I R_{L}$ or $I R_{H}$ or both are binding.

In the rest of the subsection we will establish that the low type's incentive constraint, $\mathrm{IC}_{L}$, is not binding in the seller's expected revenue maximizing mechanism. In order to do this we will first analyze the seller's problem when $\mathrm{IC}_{L}$ is relaxed.

We can write the Lagrangian to the relaxed problem (when $\mathrm{IC}_{L}$ is ignored) as

$$
\begin{aligned}
\mathcal{L} & =2 R-\lambda_{L}\left(D_{L}-1\right)-\lambda_{H}\left(D_{H}-1\right) \\
& -\mu_{H}\left[D_{H}-(1-\alpha)\left(p_{L L} c_{H} y_{L L}+\left(1-p_{L L}\right) \hat{y}_{L L}\right)+\alpha\left(p_{L H} c_{H} y_{L H}+\left(1-p_{L H}\right) \hat{y}_{L H}\right)\right] \\
& -\phi_{L L}\left(p_{L L}-1 / 2\right)-\phi_{H L}\left(p_{L H}+p_{H L}-1\right)-\phi_{H H}\left(p_{H H}-1 / 2\right),
\end{aligned}
$$

where $R$ is the seller's revenue from (2), $D_{L}$ and $D_{H}$ are the left-hand sides of $\operatorname{IR}_{L}$ and $\mathrm{IR}_{H}$, respectively, $\lambda_{L}, \lambda_{H}$, and $\mu_{H}$ are the multipliers on $\mathrm{IR}_{L}, \mathrm{IR}_{H}$ and $\mathrm{IC}_{H}$, respectively, and $\phi_{L L}, \phi_{H L}$, and $\phi_{H H}$ are the multipliers on the feasibility constraints.

Lemma 2 In the optimal solution when $I C_{L}$ is relaxed we have $p_{H H}=1 / 2, p_{H L}=1$, and $p_{L H}=0$.

The proof is based on perturbations of the Lagrangian and can be found in the Appendix. This result implies that in the optimal auction, only the low type may be 
rationed - if either of the two buyers reports a high type then the good is sold efficiently. The low type is rationed if $p_{L L}<1 / 2$.

With this much preparation we are ready to prove our first main result.

Proposition 1 In the mechanism that maximizes the seller's revenue, $I R_{L}$ and $I C_{H}$ are binding and $I C_{L}$ is slack.

Proof. We will argue that in the solution where $\mathrm{IC}_{L}$ is neglected the incentive compatibility constraint for the low type is satisfied ( $\mathrm{IC}_{L}$ holds strictly), therefore $\mathrm{IC}_{L}$ must be slack in the solution. This implies that $\mathrm{IR}_{L}$ is binding (by Lemma 1 ) and that $\mathrm{IC}_{H}$ is also binding (otherwise the first-best would be feasible).

In the solution where $\mathrm{IC}_{L}$ is neglected, we know that $p_{H H}=1 / 2, p_{H L}=1$, and $p_{L H}=0$. Moreover, in the proof of Lemma 2 we found that the high type is insured against the loss of the good, $c_{H} y_{H L}=c_{H} y_{H H}=\hat{y}_{H H} \equiv K$, and by $\mathrm{IR}_{H}, K \leq 1$.

Consider as a candidate mechanism a contract where the low type is offered complete insurace such that $\mathrm{IR}_{L}$ is binding, $c_{L} y_{L L}=\hat{y}_{L H}=\hat{y}_{L L}=1$, and $K$ is set so that the high type's incentive constraint binds,

$$
K=(1-\alpha)\left(p_{L L} c+1-p_{L L}\right)+\alpha,
$$

where $c=c_{H} / c_{L}$. Since $c<1$, we have $K<1$, therefore $\operatorname{IR}_{H}$ is slack. Since $p_{L L} \leq 1 / 2$, we also have $K \geq(c+1) / 2$.

We will now show that the candidate mechanism is feasible, that is, $\mathrm{IC}_{L}$ holds. The left-hand side of $\mathrm{IC}_{L}$ equals 1, while the right-hand side can be written as

$$
r h s\left(\mathrm{IC}_{L}\right)=\alpha \frac{K}{c}+\frac{(1-\alpha)}{2}\left(\frac{K}{c}+K\right)
$$

At $\alpha=0$, this expression becomes $(K / c+K) / 2$, which, by $K \geq(c+1) / 2$, is weakly 
greater than

$$
\frac{1}{2}\left(\frac{c+1}{2} \frac{1}{c}+\frac{c+1}{2}\right)=\frac{1}{2}\left(1+\frac{1 / c+c}{2}\right)>1 .
$$

That is, $\mathrm{IC}_{L}$ holds as a strict inequality at $\alpha=0$. However,

$$
\frac{\partial r h s\left(\mathrm{IC}_{L}\right)}{\partial \alpha}=\frac{1}{2}\left(\frac{K}{c}-K\right)>0
$$

Therefore $\mathrm{IC}_{L}$ holds strictly for all $\alpha \in[0,1]$. The candidate mechanism indeed satisfied all (incentive, participation, and feasibility) constraints.

Starting from the candidate mechanism (both types fully insured, $\mathrm{IR}_{L}$ and $\mathrm{IC}_{H}$ binding, $K<1$ ), the optimal mechanism is obtained by distorting the low type's full insurance. In order to ensure his participation, the low type's expected payment must be reduced. Then, the only way to increase the seller's revenue is by requiring the high type to pay more in the optimal mechanism than in the candidate one. This means that a low type imitating the high type would pay uniformly more in the optimal mechanism than he would have in the candidate one, therefore $\mathrm{IC}_{L}$ must hold as a strict inequality in the optimal mechanism as well.

Remark 1 It is clear from the proof of Proposition 1 that the same result holds for the expected revenue maximizing efficient auction as well (i.e., when $p_{L L}=1 / 2$ instead of being set optimally).

There are three types of risk that the buyers may face in the optimal auction. First, their payments conditional on the type-announcements and the allocation could be random - this is excluded by assuming constant absolute risk aversion. Second, for a given allocation, a buyer may face risk arising from the other buyer's type announcement. Third, for a given type-profile, a buyer's marginal utility of income may be 
different when he wins and when he loses. ${ }^{3}$ In the optimal auction, as we have shown, the high type faces no risk of the second kind because $y_{H H}=y_{H L}$, and he is insured against risk of the third kind because $c_{H} y_{H H}=\hat{y}_{H H}$. On the other hand, the low type almost always incurs both types of risks. Only if the buyers types are independent and he does not win will the low type's marginal utility of income be independent of the other buyer's valuation.

The significance of Proposition 1 is that it shows that our optimal auction design problem is "regular" in the sense that in the optimum the low type's incentive compatibility constraint is not binding (while the high type's is), and the low type enjoys no rents. (The proof is different from that in the risk neutral case because of interesting insurance considerations.) In the next subsection we will show that when the buyers' types are sufficiently strongly correlated, neither type earns any information rents in the optimal auction.

\subsection{Full Rent Extraction in the Optimal Auction}

We have established that $\mathrm{IR}_{L}$ and $\mathrm{IC}_{H}$ are binding, and $\mathrm{IC}_{L}$ is slack in the optimal auction. Whether $\mathrm{IR}_{H}$ is binding (whether the high type has no surplus either), it turns out, depends on how strong the correlation between the types is. Recall that in our model, the correlation coefficient between high and low types is $2 \alpha-1$.

Proposition 2 If the correlation between high and low valuations is greater than $1 / 3$ in absolute value (i.e., $\alpha \notin[1 / 3,2 / 3]$ ), then no matter how risk-averse the buyers are (i.e., for all $r>0$ ), in the optimal auction neither type of the buyers earns any surplus, that is, both $I R_{L}$ and $I R_{H}$ are binding.

\footnotetext{
${ }^{3}$ The latter possibility arises because ties happen with positive probability in a discrete-type model.
} 
Proof. Suppose that $\mathrm{IR}_{H}$ is slack. Then $\lambda_{H}=0$ in the Lagrangian, and the KuhnTucker conditions of maximization with respect to the $y$-variables become

$$
\begin{aligned}
\frac{\partial \mathcal{L}}{\partial y_{L L}} & =\frac{\alpha}{r} \frac{p_{L L}}{y_{L L}}-\lambda_{L} \alpha p_{L L} c_{L}+\mu_{H}(1-\alpha) p_{L L} c_{H}=0, \\
\frac{\partial \mathcal{L}}{\partial \hat{y}_{L L}} & =\frac{\alpha}{r} \frac{1-p_{L L}}{\hat{y}_{L L}}-\lambda_{L} \alpha\left(1-p_{L L}\right)+\mu_{H}(1-\alpha)\left(1-p_{L L}\right)=0, \\
\frac{\partial \mathcal{L}}{\partial \hat{y}_{L H}} & =\frac{1-\alpha}{r} \frac{1}{\hat{y}_{L H}}-\lambda_{L}(1-\alpha)+\mu_{H} \alpha=0, \\
\frac{\partial \mathcal{L}}{\partial y_{H H}} & =\frac{\alpha}{r} \frac{1 / 2}{y_{H H}}-\mu_{H} \alpha \frac{1}{2} c_{H}=0, \\
\frac{\partial \mathcal{L}}{\partial \hat{y}_{H H}} & =\frac{\alpha}{r} \frac{1 / 2}{\hat{y}_{H H}}-\mu_{H} \frac{1}{2} \alpha=0, \\
\frac{\partial \mathcal{L}}{\partial y_{H L}} & =\frac{1-\alpha}{r} \frac{1}{y_{H L}}-\mu_{H}(1-\alpha) c_{H}=0 .
\end{aligned}
$$

Cross-multiplying the first three equations by $y_{L L}, \hat{y}_{L L}$, and $\hat{y}_{L H}$, respectively, and adding them up yields

$$
\begin{aligned}
\frac{1}{r}=\left[\alpha p_{L L} c_{L} y_{L L}+\alpha\left(1-p_{L L}\right) \hat{y}_{L L}+(1-\alpha) \hat{y}_{L H}\right] \lambda_{L} \\
\\
\quad-\left[(1-\alpha) p_{L L} c_{H} y_{L L}+(1-\alpha)\left(1-p_{L L}\right) \hat{y}_{L L}+\alpha \hat{y}_{L H}\right] \mu_{H} .
\end{aligned}
$$

From the second three equations,

$$
\frac{1}{r \mu_{H}}=c_{H} y_{H H}=c_{H} y_{H L}=\hat{y}_{H H} .
$$

Note that $D_{H} \equiv 1 / r \mu_{H}$, hence $1 / r \mu_{H} \leq 1$. The high type's incentive constraint is binding because $\mathrm{IR}_{H}$ is slack. Hence $\mathrm{IC}_{H}$ can be written as

$$
\frac{1}{r \mu_{H}}=(1-\alpha)\left(p_{L L} c_{H} y_{L L}+\left(1-p_{L L}\right) \hat{y}_{L L}\right)+\alpha \hat{y}_{L H} .
$$


In equation (4), the coefficient on $\lambda_{L}$ equals 1 by $\mathrm{IR}_{L}$, and the coefficient on $\mu_{H}$ equals $-1 / r \mu_{H}$ by $\mathrm{IC}_{H}$. Therefore $\lambda_{L}=2 / r$.

From the second and third Kuhn-Tucker conditions used above,

$$
\begin{aligned}
\hat{y}_{L L} & =\frac{\alpha}{r \lambda_{L} \alpha-r \mu_{H}(1-\alpha)}, \\
\hat{y}_{L H} & =\frac{1-\alpha}{r \lambda_{L}(1-\alpha)-r \mu_{H} \alpha} .
\end{aligned}
$$

Recall $\lambda_{L}=2 / r$ and $r \mu_{H} \geq 1$. If $\alpha<1 / 3$ then the denominator in $\hat{y}_{L L}, r \lambda_{L} \alpha-r \mu_{H}(1-$ $\alpha) \leq 2 \alpha-(1-\alpha)=3 \alpha-1$, is negative, so $\hat{y}_{L L}<0$, a contradiction. If $\alpha>2 / 3$ then the denominator in $\hat{y}_{L H}, r \lambda_{L}(1-\alpha)-r \mu_{H} \alpha \leq 2(1-\alpha)-\alpha=2-3 \alpha$, is negative, so $\hat{y}_{L H}<0$, a contradiction. Therefore, if $\mathrm{IR}_{H}$ is slack then $\alpha$ cannot lie outside the interval $[1 / 3,2 / 3]$.

Proposition 2 shows that the well-known property of optimal auctions under correlation and risk neutrality (Crémer-McLean mechanisms) that they leave no surplus with the buyers is not so peculiar: even if the buyers are risk-averse (in fact, for any level of constant absolute risk aversion), the seller's optimal mechanism exhibits the same feature provided the correlation of valuations is sufficiently strong. ${ }^{4}$

An interesting comparative statics result is that for $\alpha \neq 1 / 2$ (with valuations that are not independent), the seller's expected revenue is not monotonic in $r$ (the buyers' risk-aversion parameter)..$^{5}$ This is so because in both limits, as $r \rightarrow 0$ and as $r \rightarrow \infty$, the seller's revenue converges to the first-best. Intuitively, the non-monotonicity is the result of two effects. Start with risk-neutral buyers and correlated values: there exists a

\footnotetext{
${ }^{4}$ Note, however, that the seller's expected revenue is not equal to the first-best when the buyers are risk-averse.

${ }^{5}$ In contrast, in the independent-values case Matthews [5] shows that in the optimal auction the seller's revenue is increasing with $r$.
} 
family of full-rent extracting mechanisms. As $r$ increases, it becomes more costly for the seller to compensate the buyers for the risks of any of these mechanisms. However, other auction forms, such as the first-price auction, which exploit the buyers' risk aversion, become more profitable. As $r$ becomes sufficiently high, these auctions become optimal.

Another observation is that the outcome of the optimal auction is risky not only for the buyers but the seller as well. Consequently, one may ask how to design an optimal auction when the seller is risk-averse. In the classical setup (independent private values, risk neutral buyers) Eső and Futó [3] show that the seller can get full insurance against the revenue-risk of any auction at no cost. Therefore, in each expected-revenue equivalence class there is a mechanism with a deterministic revenue, which is optimal for the risk-averse seller. However, when the buyers' valuations are correlated, there may be a trade-off between the expected value and the riskiness of the seller's revenue.

To see this point, consider our model with binary types, positive correlation $(\alpha>$ $1 / 2)$, and risk-neutral buyers $(r \rightarrow 0)$. Suppose that the allocation rule is such that the low type won't win against the high type but may be rationed $\left(p_{L L} \leq 1 / 2, p_{L H}=0\right)$, just like in the optimal auction of Propositions 1 and 2. Denote the expected transfers from a buyer to the seller when he announces low and the other buyer announces low and high by $t_{L L}$ and $t_{L H}$, respectively. In any Crémer-McLean mechanism neither type enjoys any surplus. The participation constraint of the low type is

$$
\alpha\left(p_{L L} L-t_{L L}\right)-(1-\alpha) t_{L H}=0,
$$

while the high type's incentive compatibility constraint is

$$
0 \geq(1-\alpha)\left(p_{L L} H-t_{L L}\right)-\alpha t_{L H} .
$$


(The left-hand side of the latter constraint is zero because the high type makes no profit in a Crémer-McLean auction.) From the former equation, $t_{L H}=\alpha /(1-\alpha)\left(p_{L L} L-t_{L L}\right)$, which substituted into the latter inequality yields

$$
\frac{\alpha}{1-\alpha}\left(p_{L L} L-t_{L L}\right) \geq \frac{1-\alpha}{\alpha}\left(p_{L L} H-t_{L L}\right) .
$$

If $p_{L L} L<t_{L L}$ then both sides of the above inequality would be negative; buth then since $L<H$ and $\alpha /(1-\alpha)>1>(1-\alpha) / \alpha$ (because of $\alpha>1 / 2)$, the inequality would be violated. Therefore $t_{L L} \leq p_{L L} L$, so in the state of nature when both buyers report $L$ the seller's revenue does not exceed $2 p_{L L} L \leq L$. However, an ordinary English auction would yield a revenue of at least $L$ in every state of nature, which is preferred by a sufficiently risk averse seller. This shows that a sufficiency risk averse seller would not choose a full-rent-extracting mechanisms when the correlation of valuations is positive even if the buyers were risk neutral.

\section{Conclusions}

We studied the expected revenue maximizing mechanism in a simple auction environment with risk-averse buyers (CARA preferences) and correlated private values (binary types, symmetric distribution). Correlation of the valuations makes it possible for the seller to extract all rents from risk-neutral buyers (Crémer and McLean [1], [2]); however, riskaverse buyers require compensation for the risks involved in such mechanisms. On the other hand, we know from Matthews [5] and Maskin and Riley [4] that risk aversion of the buyers may also help the seller to screen them and hence it may increase the seller's expected revenue.

We found that the optimal auction with risk-averse bidders retains the full-rent- 
extracting property of Crémer-McLean mechanisms, provided that the correlation of the buyers' valuations is sufficiently strong. (In our model with CARA preferences, if the correlation is sufficiently strong then both types have zero surplus, no matter how risk-averse the buyers are.) We also showed that the same result does not hold if the seller is sufficiently risk-averse because full-rent-extracting mechanisms generate highly risky revenue for the seller even if the buyers are risk neutral.

Among other questions, the generalization of the model to more than two types and a fuller analysis of the case of a risk-averse seller may be interesting for future research.

\section{Appendix: Omitted Proofs}

Proof of Lemma 1. In order to prove the first claim, suppose towards contradiction that both $\mathrm{IR}_{i}$ and $\mathrm{IC}_{i}$ are slack. Then, the seller can increase one of the variables $y_{i j}$ or $\hat{y}_{i j}$ that appears with a positive coefficient in $D_{i}$ by $\varepsilon>0$. For a sufficiently small $\varepsilon$ both $\mathrm{IR}_{i}$ and $\mathrm{IC}_{i}$ continue to hold while the other IR and IC constraints are relaxed and the seller's revenue increases, which is a contradiction.

Second, suppose towards contradiction that both $\mathrm{IR}_{L}$ or $\mathrm{IR}_{H}$ are slack. Increase $\hat{y}_{L L}$ by $\varepsilon /\left(1-p_{L L}\right)>0$ (note $p_{L L} \leq 1 / 2$ by symmetry and feasibility). This change increases $D_{L}$ (the left-hand sides of both $\mathrm{IR}_{L}$ and $\mathrm{IC}_{L}$ ) by $\alpha \varepsilon$ and the right-hand side of $\mathrm{IC}_{H}$ by $(1-\alpha) \varepsilon$. Also, increase either $y_{H L}$ by $\varepsilon /\left(c_{H} p_{H L}\right)$ (if $\left.p_{H L}>0\right)$, or $\hat{y}_{H L}$ by $\varepsilon$ (if $\left.p_{H L}=0\right)$.

This increases $D_{H}$ (the left-hand sides of $\mathrm{IR}_{H}$ and $\mathrm{IC}_{H}$ ) by $(1-\alpha) \varepsilon$ and the right-hand side of $\mathrm{IC}_{L}$ by at least $\alpha \varepsilon$ ( since $c_{H}<c_{L}$ ). For $\varepsilon>0$ sufficiently small this perturbation is feasible, and the seller's revenue increases, which is a contradiction.

Proof of Lemma 2. First, we claim that in the solution $p_{H H}>0$. Suppose towards contradiction that $p_{H H}=0$. Increase $p_{H H}$ to $\varepsilon>0$ and set $y_{H H}=\hat{y}_{H H} / c_{H}$. No 
constraint is affected as $D_{H}$, the left-hand sides of $\mathrm{IR}_{H}$ and $\mathrm{IC}_{H}$, is unchanged. The seller's revenue, $2 R$, changes by the amount $\alpha \varepsilon\left(\ln y_{H H}-\ln \hat{y}_{H H}\right) / r$, which is positive by $c_{H}<1$, a contradiction.

Now we will show $p_{H H}=1 / 2$. The necessary Kuhn-Tucker conditions of maximization with respect to $y_{H H}$ and $\hat{y}_{H H}$ are

$$
\begin{aligned}
\frac{\partial \mathcal{L}}{\partial y_{H H}} & =\frac{\alpha}{r} \frac{p_{H H}}{y_{H H}}-\left(\lambda_{H}+\mu_{H}\right) \alpha p_{H H} c_{H}=0, \\
\frac{\partial \mathcal{L}}{\partial \hat{y}_{H H}} & =\frac{\alpha}{r} \frac{1-p_{H H}}{\hat{y}_{H H}}-\left(\lambda_{H}+\mu_{H}\right) \alpha\left(1-p_{H H}\right)=0 .
\end{aligned}
$$

(Note that the first-order conditions with respect to $y_{i j}$ hold as equalities because $y_{i j}>$ 0.) From $p_{H H} \in(0,1)$ we get $c_{H} y_{H H}=\hat{y}_{H H}=1 /\left(r \lambda_{H}+r \mu_{H}\right)$. Moreover, by the Kuhn-Tucker conditions with respect to $p_{H H}$ and $\phi_{H H}$,

$$
\begin{aligned}
\frac{\partial \mathcal{L}}{\partial p_{H H}} & =\frac{\alpha}{r}\left(\ln y_{H H}-\ln \hat{y}_{H H}\right)-\left(\lambda_{H}+\mu_{H}\right) \alpha\left(c_{H} y_{H H}-\hat{y}_{H H}\right)-\phi_{H H}=0, \\
-\frac{\partial \mathcal{L}}{\partial \phi_{H H}} & =p_{H H}-\frac{1}{2} \leq 0, \phi_{H H} \geq 0, \text { and }\left(p_{H H}-\frac{1}{2}\right) \phi_{H H}=0 .
\end{aligned}
$$

$\left(\partial \mathcal{L} / \partial p_{H H}=0\right.$ because $\left.p_{H H}>0\right)$. Using $c_{H} y_{H H}=\hat{y}_{H H}$ the former condition becomes $\alpha \ln \left(1 / c_{H}\right) / r=\phi_{H H}$, hence $\phi_{H H}>0$. But then by the latter condition $p_{H H}=1 / 2$ as claimed.

In order to show that in the optimum $p_{H L}=1$ and $p_{L H}=0$, first fix any $p_{L H}, p_{H L} \in$ $(0,1)$ with $p_{L H}+p_{H L} \leq 1$, and choose $y_{H L}, \hat{y}_{H L}, y_{L H}$, and $\hat{y}_{L H}$ optimally. The KuhnTucker conditions with respect to $y_{H L}$ and $\hat{y}_{H L}$ are,

$$
\begin{aligned}
& \frac{\partial \mathcal{L}}{\partial y_{H L}}=\frac{1-\alpha}{r} \frac{p_{H L}}{y_{H L}}-\left(\lambda_{H}+\mu_{H}\right)(1-\alpha) p_{H L} c_{H}=0, \\
& \frac{\partial \mathcal{L}}{\partial \hat{y}_{H L}}=\frac{1-\alpha}{r} \frac{1-p_{H L}}{\hat{y}_{H L}}-\left(\lambda_{H}+\mu_{H}\right)(1-\alpha)\left(1-p_{L H}\right)=0 .
\end{aligned}
$$


The two conditions imply $c_{H} y_{H L}=\hat{y}_{H L}$. Similarly, the Kuhn-Tucker conditions with respect to $y_{L H}$ and $\hat{y}_{L H}$ are,

$$
\begin{aligned}
\frac{\partial \mathcal{L}}{\partial y_{L H}} & =\frac{1-\alpha}{r} \frac{p_{L H}}{y_{L H}}-\lambda_{L}(1-\alpha) p_{L H} c_{L}+\mu_{H} \alpha p_{L H} c_{H}=0 \\
\frac{\partial \mathcal{L}}{\partial \hat{y}_{L H}} & =\frac{1-\alpha}{r} \frac{1-p_{L H}}{\hat{y}_{L H}}-\lambda_{L}(1-\alpha)\left(1-p_{L H}\right)+\mu_{H} \alpha\left(1-p_{L H}\right)=0 .
\end{aligned}
$$

If we replace $c_{L}$ by $c_{H}<c_{L}$ in the first condition then the resulting inequality and the second condition together imply $c_{H} y_{L H}<\hat{y}_{L H}$. Multiplying the original conditions by $y_{L H} / p_{L H}$ and $-\hat{y}_{L H} /\left(1-p_{L H}\right)$, respectively, and adding them up yields,

$$
-\lambda_{L}(1-\alpha)\left(c_{L} y_{L H}-\hat{y}_{L H}\right)+\mu_{H} \alpha\left(c_{H} y_{L H}-\hat{y}_{L H}\right)=0
$$

Now consider perturbing $p_{H L}$ and $p_{L H}$ by $d p$ and $-d p$, respectively. The corresponding change in the Lagrangian is,

$$
\begin{aligned}
d \mathcal{L} & =\left[\frac{1-\alpha}{r}\left(\ln y_{H L}-\ln \hat{y}_{H L}\right)-\left(\lambda_{H}+\mu_{H}\right)(1-\alpha)\left(c_{H} y_{H L}-\hat{y}_{H L}\right)-\phi_{H L}\right] d p \\
& -\left[\frac{1-\alpha}{r}\left(\ln y_{L H}-\ln \hat{y}_{L H}\right)-\lambda_{L}(1-\alpha)\left(c_{L} y_{L H}-\hat{y}_{L H}\right)+\mu_{H} \alpha\left(c_{H} y_{L H}-\hat{y}_{L H}\right)-\phi_{H L}\right] d p .
\end{aligned}
$$

Using $\hat{y}_{H L}=c_{H} y_{H L}$ and equation $(5), d \mathcal{L}$ can be rewritten as

$$
d \mathcal{L}=\left[\frac{1-\alpha}{r} \ln \left(1 / c_{H}\right)-\frac{1-\alpha}{r}\left(\ln y_{L H}-\ln \hat{y}_{L H}\right)\right] d p .
$$

By $c_{H} y_{L H}<\hat{y}_{L H}$, the perturbation increases the Lagrangian, therefore $p_{L H}=0$. Moreover, if $p_{H L}$ is less than 1 in the optimum then $\phi_{H L}$ must be zero (by complementary slackness), and hence $\partial \mathcal{L} / \partial p_{H L}=(1-\alpha) \ln \left(1 / c_{H}\right) / r>0$. Therefore $p_{H L}=1$. 


\section{References}

[1] J. Crémer, R. P. McLean, Optimal Selling Strategies under Uncertainty for a Discriminating Monopolist, Econometrica 53 (1985) 345-361.

[2] J. Crémer, R. P. McLean, Full Extraction of the Surplus in Bayesian and Dominant Strategy Auctions, Econometrica 56 (1988) 345-361.

[3] P. Eső, G. Futó, Auction Design with a Risk Averse Seller, Econ. Letters 65 (1999) 71-74.

[4] E. Maskin, J. Riley, Optimal Auctions with Risk Averse Buyers, Econometrica 52 (1984) 1473-1518.

[5] S. Matthews, Selling to Risk-Averse Buyers with Unobservable Tastes, J. Econ. Theory 30 (1983) 370-400.

[6] P. Milgrom, R. Weber, A Theory of Auctions and Competitive Bidding, Econometrica 50 (1982) 1089-1122.

[7] R. Myerson, Optimal Auction Design, Mathematics of Operations Research 6 (1981) 58-73.

[8] J. Riley, W. Samuelson, Optimal Auctions, Amer. Econ. Rev. 71 (1981) 381-92.

[9] W. Vickrey, Counterspeculation, Auctions, and Competitive Sealed Tenders, J. Finance 16 (1961) 8-37.

[10] K. Waehrer, R. M. Harstad, M. H. Rothkopf, Auction Form Preferences of RiskAverse Bid Takers, RAND J. Econ. 29 (1998) 179-192. 